\title{
Computational aspects of line graph of carbon nanocones
}

\author{
Zafar Hussain ${ }^{1}$, Mobeen Munir ${ }^{2}$, Waqas Nazeer ${ }^{3}$, Muhammad Shoaib Saleem ${ }^{4}$, Shin Min Kang ${ }^{5,6^{*}}$ and Young \\ Chel Kwun ${ }^{7}$ \\ ${ }^{1}$ Department of Mathematics and Statistics, University of Lahore, Lahore, Pakistan. \\ ${ }^{2}$ Department of Mathematics Division of Science and Technology, University of Education, Lahore, 54000, Pakistan. \\ ${ }^{3}$ Department of Mathematics, Government College University, Lahore, Pakistan. \\ ${ }^{4}$ Division of Science and Technology, University of Okara, Pakistan. \\ ${ }^{5}$ Department of Mathematics and RINS, Gyeongsang National University, Jinju, South Korea. \\ ${ }^{6}$ Center for General Education, China Medical University, Taiwan, Taichung, Taiwan \\ ${ }^{7}$ Department of Mathematics, Dong-A University, Busan, South Korea.
}

Submitted: 24 March 2017; Revised: 23 September 2018; Accepted: 23 August 2019

\begin{abstract}
Graph theory plays substantial role in mathematics, chemistry, QSAR and physical sciences. The basic layout of the graph theoretic model is a molecular structure in which vertices of the graph correspond to atoms, and edges correspond to chemical bonds. The study of this graph model provides information about the chemical structure. A line graph has many useful applications in physical chemistry. M-polynomial is rich in producing closed forms of many degree-based topological indices which correlates chemical properties of the material under investigation. This polynomial is used in computing closed formulas of many degree-based topological invariants of the molecular structures. The molecular graph of carbon nanocones has a conical structure with a cycle of length $\mathrm{k}$ at its core and $n$ layers of hexagons placed at the conical surface around its centre. In this study, we transformed the molecular structure of carbon nanocones into graph theoretic model and produced its line graph. Thereafter, we determined closed formulas for M-polynomials of line graphs of nanocones. We also recovered important topological degree-based indices of the line graph of nanocones. Moreover, we provide different graphs of topological indices and their relations with the parameters of the line graph of nanocones. These graphs depict the actual dependencies of the topological indices on the parameters of the carbon nanocones.
\end{abstract}

Keywords: Degree-based topological index, general Randic index, line graph, M-polynomial, symmetric division index, Zagreb index.

\section{INTRODUCTION}

In mathematical chemistry, mathematical tools such as polynomials and numbers predict properties of compounds without using quantum mechanics. These tools, in combination, capture information hidden in the symmetry of molecular graphs. Topological indices are numerical parameters of a graph, which characterise its topology and are usually graph invariant. They describe the structure of molecules numerically and are used in the development of qualitative structure activity relationships (QSARs). The most commonly known invariants of such kind are degree-based topological indices. These are actually the numerical values that correlate the structure with various physical properties, chemical reactivity, and biological activities (Klavžar \& Gutman, 1996; Rucker \& Rucker, 1999; Brückler et al., 2011). It is an established fact that many properties such as heat of formation, boiling point, strain energy, rigidity and fracture toughness of a molecule are strongly connected to its graphical structure and this fact plays a synergic role in chemical graph theory.

Several algebraic polynomials have useful applications in chemistry such as Hosoya polynomial (also called Wiener polynomial) (Gutman, 1993),

*Corresponding author (Smkang@gnu.ac.kr; (iD https://orcid.org/0000-0002-5294-4447) 
which play a vital role in determining distance-based topological indices. Among other algebraic polynomials, M-polynomial (Deutsch \& Klavzar, 2015) introduced in 2015 , plays the same role in determining the closed form of many degree-based topological indices (Munir et al. 2016a, b, c, d, 2017; Kwun et al., 2017; Ali et al., 2018). The main advantage of M-polynomial is the wealth of information that it contains about degree-based graph invariants.

Carbon nanocones have been observed since 1968 or even earlier (Gillot et al., 1968) on the surface of naturally occurring graphite. Their bases are attached to the graphite and the height varies between 1 to 40 micrometers. The walls are often curved and are less regular than those of the laboratory made nanocones. Carbon nanostructures have attracted considerable attention due to their potential uses in many applications including energy storage, gas sensors, biosensors, nanoelectronic devices and chemical probes (Iijima, 1991). Carbon allotropes such as carbon nanocones and carbon nanotubes have been proposed as possible molecular gas storage devices (Zhao et al., 2002; Adisa et al., 2011). More recently, carbon nanocones have gained increased scientific interests due to their unique properties and promising uses in many novel applications such as energy and gas storage. Figure 1 depicts the structure of a carbon nanocone.

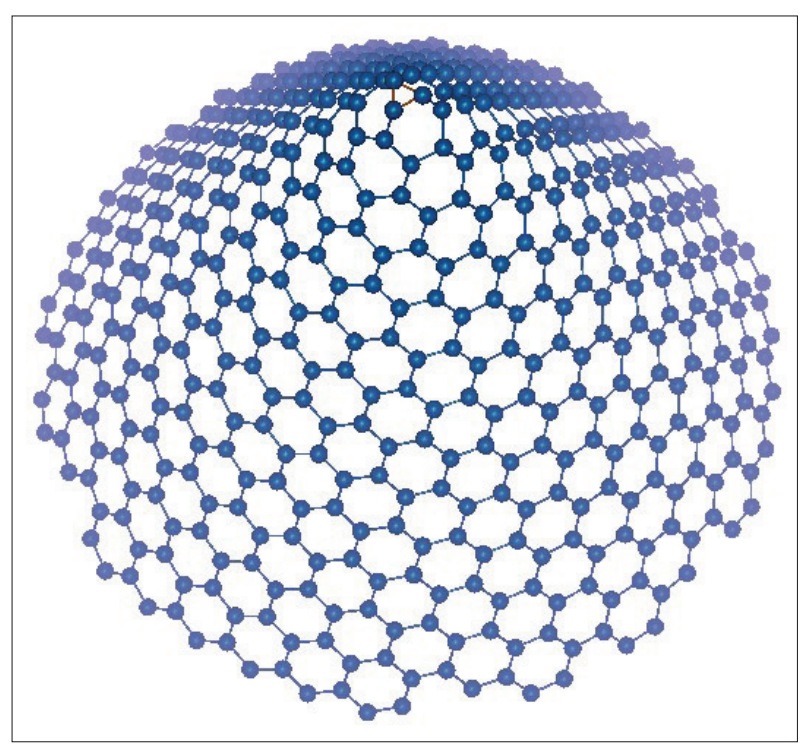

Figure 1: Carbon nanocone $C N C_{k}[n]$
The molecular graph of $C N C_{k}[n]$ nanocones has a conical structure with a cycle of length $\mathrm{k}$ at its core and $n$ layers of hexagons placed at the conical surface around its centre as shown in Figure 2.

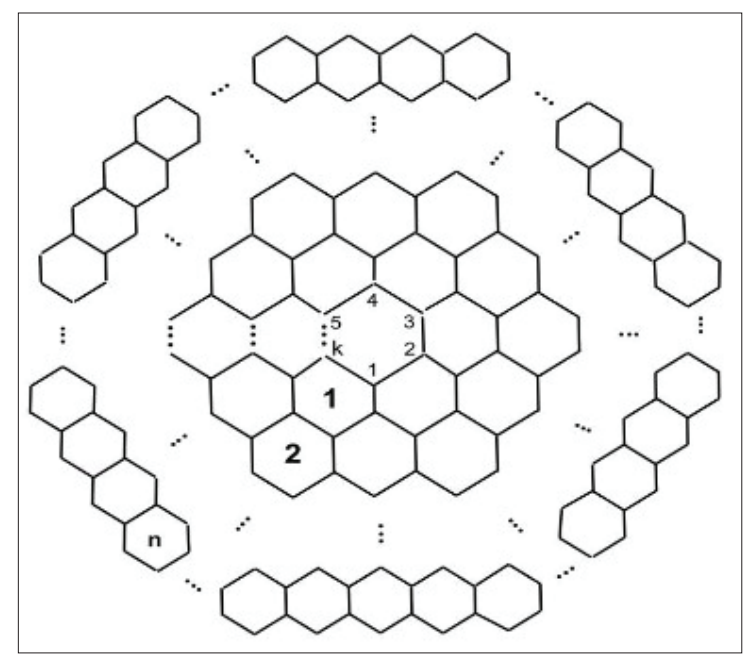

Figure 2: Carbon nanocones $C N C_{k}[n]$

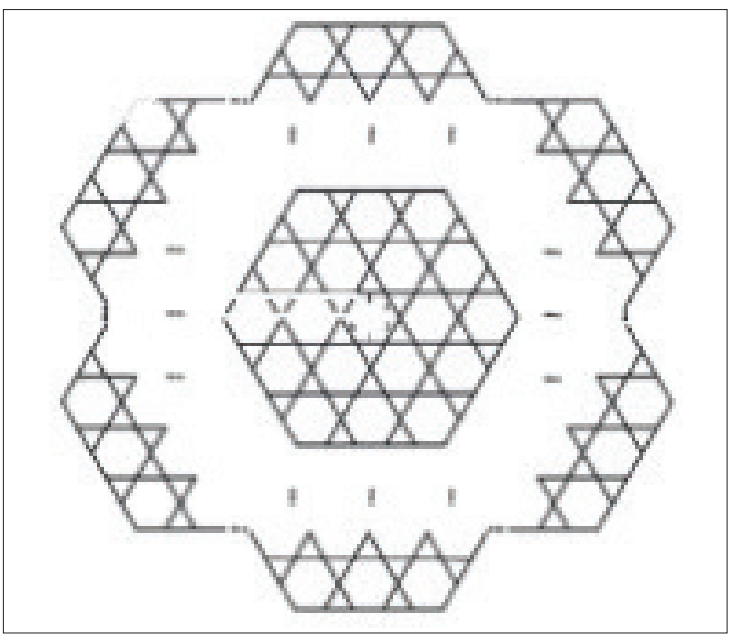

Figure 3: Line graph of the carbon nanocones $\mathrm{CNC}_{k}[n]$

The line graph $L(G)$ of a graph $G$ is the graph each of whose vertices represents an edge of $G$ and two of its vertices are adjacent if their corresponding edges are adjacent in $G$ (Iranmanesh et al., 2009; Huo et al., 2016). In Figure 3, we can see the line graph of the carbon nanocones $C N C_{k}[n]$. 
The present paper is concerned with the line graph of the carbon nanocone $C N C_{k}[n]$ where $k$ is the number of hexagons forming the base of the cone and $n$ is the number of layers of the cone. In Wu et al. (2010), authors computed the Wiener index of a graph defined as $W(G)=\sum_{\{u, v\} \subset V(G)} d_{G}(u, v)$. They also computed the Wiener index of the line graph and established new bounds on the Wiener index of the line graph of any graph. In Buckley and Rapoport (1981), authors computed mean distances in line graphs. In Cataldo et al. (2010), Wiener index was used as pure topological potential energy of graphenic infinite structures producing interesting results, assigning to fullerene-like nanostructures a nonnegligible probability of formation on graphene sheets on carbon black or on amorphous carbon in general. Authors also showed that 1-pentagonal nanocones become chemically reactive when it reaches certain sizes. Ashrafi and Mohammad-Abadi (2012) computed Weiner index of 1-pentagonal nanocones.

In this paper, we compute M-polynomial and degreebased topological indices of the line graph of nanocones. We also give many degree-based topological indices in closed forms for the line graphs of nanocones.

\section{METHODOLOGY, RESULTS AND DISCUSSION}

Throughout this paper, $\mathrm{G}$ is a connected graph, $V(G)$ and $E(G)$ are the vertex set and edge set, respectively and $d_{v}$ denotes the degree of a vertex $v$.

Definition 1. (Deutsch \& Klavzar, 2015). The M-polynomial of $G$ is defined as:

$$
M(G, x, y)=\sum_{\delta \leq i \leq j \leq \Delta} m_{i j}(G) x^{i} y^{j}
$$

where $\delta=\operatorname{Min}\left\{d_{v} \mid v \in \mathrm{V}(\mathrm{G})\right\}, \Delta=\operatorname{Max}\left\{d_{v} \mid v \in \mathrm{V}(\mathrm{G})\right\}$, and $m_{i j}(G)$ is the edge $v u \in E(G)$ such that $\left\{d_{v}, d_{u}\right\}=\{i, j\}$.

The first topological index was introduced by Wiener (1947) and it was named path number, which is now known as Wiener index. In chemical graph theory, this is the most studied molecular topological index due to its wide applications [see for details (Gutman \& Polansky, 1986; Dobrynin et al., 2001)]. Randic' index, (Randić, $1975)$ denoted by $R_{-1 / 2}(G)$ and introduced by Milan Randić in 1975 is also one of the oldest topological indices. The Randić index is defined as:
$R_{-1 / 2}(G)=\sum_{u v \in E(G)} \frac{1}{\sqrt{d_{u} d_{v}}}$.

Working independently, Bollobas and Erdos (1998) and Amić et al. (1998) proposed the generalised Randic' index and it has been studied extensively by both chemists and mathematicians (Hu et al., 2005). Many mathematical properties have been discussed (Caporossi et al., 2003). For a detailed survey, we refer the book (Li et al., 2006).

The general Randić index is defined as:

$R_{\alpha}(G)=\sum_{u v \in E(G)}\left(d_{u} d_{v}\right)^{\alpha}$, and the inverse Randic' index is defined as $R R_{\alpha}(G)=\sum_{u v \in E(G)} \frac{1}{\left(d_{u} d_{v}\right)^{\alpha}}$. Here $\alpha$ can be any real number. Obviously $R_{-1 / 2}(G)$ is the particular case of $R_{\alpha}(G)$ when $\alpha=-\frac{1}{2}$.

The Randić index is the most popular, most often applied and most studied among all other topological indices. Many papers and books such as Kier and Hall $(1976 ; 1986)$ are written on this topological index. Randić himself wrote two reviews on his Randic index (Randić, $2001 ; 2008$ ) and there are more reviews (for example Li $\&$ Shi, 2008). The suitability of the Randić index for drug design was immediately recognised, and eventually, the index was used for this purpose on countless occasions. The physical reason for the success of such a simple graph invariant is still an enigma, although several moreor-less plausible explanations were offered.

Gutman and Trinajstic' (1972) introduced the first Zagreb index and second Zagreb index, which are defined as: $M_{1}(G)=\sum_{u v \in E(G)}\left(d_{u}+d_{v}\right)$ and $M_{2}(G)=\sum_{u v \in E(G)}\left(d_{u} \times d_{v}\right)$, respectively. The second modified Zagreb index is defined as:

${ }^{m} M_{2}(G)=\sum_{u v \in E(G)} \frac{1}{d(u) d(v)}$.

For details about these indices, we refer Nikolić et al., (2003), Gutman and Das (2004) and Zhou and Trinajstić (2010) to the readers.

The symmetric division index is defined as:

$\operatorname{SDD}(\mathrm{G})=\sum_{u v E(G)}\left\{\frac{\min \left(d_{u}, d_{v}\right)}{\max \left(d_{u}, d_{v}\right)}+\frac{\max \left(d_{u}, d_{v}\right)}{\min \left(d_{u}, d_{v}\right)}\right\}$. 
Another variant of Randic index is the harmonic index defined as:

$$
H(G)=\sum_{v u \in E(G)} \frac{2}{d_{u}+d_{v}} .
$$

The Inverse sum-index is defined as:

$$
I(G)=\sum_{v u \in E(G)} \frac{d_{u} d_{v}}{d_{u}+d_{v}} .
$$

The augmented Zagreb index is defined as:

$$
A(G)=\sum_{v u \in E(G)}\left\{\frac{d_{u} d_{v}}{d_{u}+d_{v}-2}\right\}^{3},
$$

and it is useful for computing heat of formation of alkanes. [For details about these topological indices we refer (Rehman et al., 2017; Sardar et al., 2017; De, 2018)].

Table 1 relates some well-known degree-based topological indices with M-polynomial (Deutsch \& Klavzar, 2015).

where

$$
\begin{aligned}
& D_{x}=x \frac{\partial(f(x, y)}{\partial x}, \\
& D_{y}=y \frac{\partial(f(x, y)}{\partial y}, \\
& S_{x}=\int_{0}^{x} \frac{f(t, y)}{t} d t, \\
& S_{y}=\int_{0}^{y} \frac{f(x, t)}{t} d t, \\
& J(f(x, y))=f(x, x), \\
& Q_{\alpha}(f(x, y))=x^{\alpha} f(x, y) .
\end{aligned}
$$

A graph uses points connected by lines to show how something changes in value (as time goes by, or as something else happens). Line graph of the carbon nanocone is shown in figure 3. Let $G=L\left(C N C_{k}[n]\right)$ be the line graph of carbon nanocones $C N C_{k}[n]$. From Figure 3, we see that the graph has $8 k+2 k n$ number of vertices and $k(n+1)(3 n+1)$ number of edges. The vertex partition and edge partition of graph $\mathrm{G}$ are shown in Tables 1 and 2, respectively.

Let $G=L\left(C N C_{k}[n]\right)$ be the line graph of the

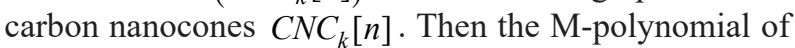

$L\left(C N C_{k}[n]\right)$ is,

$M\left(L\left(C N C_{k}[n]\right) ; x, y\right)=2 k x^{2} y^{3}+k(2 n-1) x^{3} y^{3}+2 k n x^{3} y^{4}$

$+3 k n^{2} x^{4} y^{4}$.

\section{Proof}

Let $L\left(C N C_{k}[n]\right)$ be the line graph of the carbon nanocones $C N C_{k}[n]$. It is easy to see from Figure 3 that,

$\left|V\left(L\left(C N C_{k}[n]\right)\right)\right|=8 k+2 k n$

$\left|E\left(L\left(C N C_{k}[n]\right)\right)\right|=k(n+1)(3 n+1)$

From Table 2, the vertex set of $L\left(C N C_{k}[n]\right)$ have three partitions:

$V_{1}\left(L\left(C N C_{k}[n]\right)\right)=\left\{u \in V\left(L\left(C N C_{k}[n]\right)\right): \quad d_{u}=2\right\}$,

$V_{2}\left(L\left(C N C_{k}[n]\right)\right)=\left\{u \in V\left(L\left(C N C_{k}[n]\right)\right): d_{u}=3\right\}$,

$V_{3}\left(L\left(C N C_{k}[n]\right)\right)=\left\{u \in V\left(L\left(C N C_{k}[n]\right)\right): \quad d_{u}=4\right\}$,

such that,

$\left|V_{1}\left(L\left(C N C_{k}[n]\right)\right)\right|=k$,

$\left|V_{2}\left(L\left(C N C_{k}[n]\right)\right)\right|=2 k n$,

$\left|V_{3}\left(L\left(C N C_{k}[n]\right)\right)\right|=7 k$.

From Table 3, the edge set of $L\left(C N C_{k}[n]\right)$ have four partitions:

$E_{1}\left(L\left(C N C_{k}[n]\right)\right)=\left\{e=u v \in E\left(L\left(C N C_{k}[n]\right)\right): \quad d_{u}=2, d_{v}=3\right\}$,

$E_{2}\left(L\left(C N C_{k}[n]\right)\right)=\left\{e=u v \in E\left(L\left(C N C_{k}[n]\right)\right): \quad d_{u}=d_{v}=3\right\}$,

$E_{3}\left(L\left(C N C_{k}[n]\right)\right)=\left\{e=u v \in E\left(L\left(C N C_{k}[n]\right)\right): \quad d_{u}=3,=d_{v}=4\right\}$,

$E_{4}\left(L\left(C N C_{k}[n]\right)\right)=\left\{e=u v \in E\left(L\left(C N C_{k}[n]\right)\right): \quad d_{u}=d_{v}=4\right\}$,

such that,

$\left|E_{1}\left(L\left(C N C_{k}[n]\right)\right)\right|=2 k$,

$\left|E_{2}\left(L\left(C N C_{k}[n]\right)\right)\right|=k(2 n-1)$,

$\left|E_{3}\left(L\left(C N C_{k}[n]\right)\right)\right|=2 k n$,

$\left|E_{4}\left(L\left(C N C_{k}[n]\right)\right)\right|=3 k n^{2}$, 
Table 1: Derivation of some degree-based topological indices from M-polynomial

\begin{tabular}{ll}
\hline Topological index & Derivation from $M(G ; x, y)$ \\
\hline First Zagreb & $\left(D_{x}+D_{y}\right)(M(G ; x, y))_{x=y=1}$ \\
Second Zagreb & $\left(D_{x} D_{y}\right)(M(G ; x, y))_{x=y=1}$ \\
Second modified Zagreb & $\left(S_{x} S_{y}\right)(M(G ; x, y))_{x=y=1}$ \\
Inverse Randic $\alpha \in N$ & $\left(D_{x}^{\alpha} D_{y}^{\alpha}\right)(M(G ; x, y))_{x=y=1}$ \\
General Randic $\mathbf{c} \alpha \in \mathbf{N} \alpha \in N$ & $\left(S_{x}^{\alpha} S_{y}^{\alpha}\right)(M(G ; x, y))_{x=y=1}$ \\
Symmetric division index & $\left(D_{x} S_{y}+S_{x} D_{y}\right)(M(G ; x, y))_{x=y=1}$ \\
Harmonic index & $2 S_{x} J(M(G ; x, y))_{x=1}$ \\
Inverse sum index & $S_{x} J D_{x} D_{y}(M(G ; x, y))_{x=1}$ \\
Augmented Zagreb index & $S_{x}{ }^{3} Q_{-2} J D_{x}{ }^{3} D_{y}{ }^{3}(M(G ; x, y))_{x=1}$ \\
\hline
\end{tabular}

Table 2: The partition of $V(G)$ of $G=L\left(C N C_{k}[n]\right)$

\begin{tabular}{cccc}
\hline$d_{v}$ & 2 & 3 & 4 \\
\hline Number of vertices & $\mathrm{k}$ & $2 \mathrm{kn}$ & $7 \mathrm{k}$ \\
\hline
\end{tabular}

Now from the definition of the M-polynomial,

$$
\begin{aligned}
M( & \left.L\left(C N C_{k}[n]\right) ; x, y\right)=\sum_{i \leq j} m_{i j} x^{i} y^{j} \\
= & \sum_{2 \leq 3} m_{23} x^{2} y^{3}+\sum_{3 \leq 3} m_{33} x^{3} y^{3}+\sum_{3 \leq 4} m_{34} x^{3} y^{4}+\sum_{4 \leq 4} m_{44} x^{4} y^{4} \\
= & \sum_{u v \in E_{1}\left(L\left(C N C_{k}[n]\right)\right)} m_{23} x^{2} y^{3}+\sum_{u v \in E_{2}\left(L\left(C N C_{k}[n]\right)\right)} m_{33} x^{3} y^{3} \\
& +\sum_{u v \in E_{3}\left(L\left(C N C_{k}[n]\right)\right)} m_{34} x^{3} y^{4}+\sum_{u v \in E_{4}\left(L\left(C N C_{k}[n]\right)\right)} m_{44} x^{4} y^{4} \\
= & \left|E_{1}\left(L\left(C N C_{k}[n]\right)\right)\right| x^{2} y^{3}+\left|E_{2}\left(L\left(C N C_{k}[n]\right)\right)\right| x^{3} y^{3} \\
& +\left|E_{3}\left(L\left(C N C_{k}[n]\right)\right)\right| x^{3} y^{4}+\left|E_{4}\left(L\left(C N C_{k}[n]\right)\right)\right| x^{4} y^{4} \\
= & 2 k x^{2} y^{3}+k(2 n-1) x^{3} y^{3}+2 k n x^{3} y^{4}+3 k n^{2} x^{4} y^{4} .
\end{aligned}
$$

Table 3: Edge partition of edge set of $G=L\left(C N C_{k}[n]\right)$

\begin{tabular}{lcccc}
\hline$\left(\mathrm{D}_{\mathrm{U}}, \mathrm{D}_{\mathrm{V}}\right)$ & $(2,3)$ & $(3,3)$ & $(3,4)$ & $(4,4)$ \\
\hline Number of edges & $2 \mathrm{k}$ & $\mathrm{k}(2 \mathrm{n}-1)$ & $2 \mathrm{kn}$ & $3 \mathrm{kn}^{2}$ \\
\hline
\end{tabular}

Figure 4 is plotted using Maple 13. This suggests that the values obtained by M-polynomial increase rapidly along the corners.

Now we compute some degree-based topological indices of nanocones from this M-polynomial.

\section{Proposition 2}

Let $G=L\left(C N C_{k}[n]\right)$ be the line graph of the carbon nanocones. Then,

1. $M_{1}\left(L\left(C N C_{k}[n]\right)\right)=24 k n^{2}+26 k n+4 k$.

2. $M_{2}\left(L\left(C N C_{k}[n]\right)\right)=48 k n^{2}+42 k n+3 k$.

3. ${ }^{m} M_{2}\left(L\left(C N C_{k}[n]\right)\right)=(3 / 16) k n^{2}+(7 / 18) k n+(2 / 9) k$.

4. $R R_{\alpha}\left(L\left(C N C_{k}[n]\right)\right)=3 \times 16^{\alpha} k n^{2}+2 \times 9^{\alpha} k n+2 \times 12^{\alpha} k n$ $+2 \times 6^{\alpha} k-9^{\alpha} k$. 
5. $R_{\alpha}\left(L\left(C N C_{k}[n]\right)\right)=k\left(\frac{3}{16^{\alpha}} n^{2}+\frac{2}{9^{\alpha}} n+\frac{2}{12^{\alpha}} n-\frac{1}{9^{\alpha}}+\frac{2}{6^{\alpha}}\right)$.

6. $\operatorname{SSD}\left(L\left(C N C_{k}[n]\right)\right)=6 k n^{2}+\frac{49}{6} k n+\frac{7}{3} k$.

7. $H\left(L\left(C N C_{k}[n]\right)\right)=\frac{3}{8} k n^{2}+\frac{13}{21} k n+\frac{7}{30} k$.

8. $I\left(L\left(C N C_{k}[n]\right)\right)=6 k n^{2}+\frac{45}{7} k n+\frac{9}{10} k$.

9. $A\left(L\left(C N C_{k}[n]\right)\right)=2048 k n^{2}+\frac{10557}{10} k n-\frac{153}{4} k$.

\section{Proof}

Let,

$M\left(L\left(C N C_{k}[n]\right) ; x, y\right)=f(x, y)=2 k x^{2} y^{3}+k(2 n-1) x^{3} y^{3}$

$+2 k n x^{3} y^{4}+3 k n^{2} x^{4} y^{4}$.

Then,

$D_{x}(f(x, y))=4 k x^{2} y^{3}+3 k(2 n-1) x^{3} y^{3}+6 k n x^{3} y^{4}$

$+12 k n^{2} x^{4} y^{4}$,

$D_{y}(f(x, y))=6 k x^{2} y^{3}+3 k(2 n-1) x^{3} y^{3}+8 k n x^{3} y^{4}$

$+12 k n^{2} x^{4} y^{4}$

$\left(D_{y} D_{x}\right)(f(x, y))=12 k x^{2} y^{3}+9 k(2 n-1) x^{3} y^{3}+24 k n x^{3} y^{4}$

$+48 k n^{2} x^{4} y^{4}$

$S_{x} S_{y}(f(x, y))=\frac{1}{3} k x^{2} y^{3}+\frac{1}{9} k(2 n-1) x^{3} y^{3}+\frac{1}{6} k n x^{3} y^{4}$

$+\frac{3}{16} k n^{2} x^{4} y^{4}$,

$D_{x}^{\alpha} D_{y}^{\alpha}(f(x, y))=2 \times 6^{\alpha} k x^{2} y^{3}+9^{\alpha} k(2 n-1) x^{3} y^{3}$

$+2 \times 12^{\alpha} k n x^{3} y^{4}+3 \times 16^{\alpha} k n^{2} x^{4} y^{4}$

$S_{x}^{\alpha} S_{y}^{\alpha}(f(x, y))=\frac{2}{6^{\alpha}} k x^{2} y^{3}+\frac{1}{9^{\alpha}} k(2 n-1) x^{3} y^{3}$

$+\frac{2}{12^{\alpha}} k n x^{3} y^{4}+\frac{3}{16^{\alpha}} k n^{2} x^{4} y^{4}$,
$S_{y} D_{x}(f(x, y))=\frac{4}{3} k x^{2} y^{3}+k(2 n-1) x^{3} y^{3}+\frac{3}{2} k n x^{3} y^{4}$

$+3 k n^{2} x^{4} y^{4}$

$S_{x} D_{y}(f(x, y))=3 k x^{2} y^{3}+k(2 n-1) x^{3} y^{3}+\frac{8}{3} k n x^{3} y^{4}$

$+3 k n^{2} x^{4} y^{4}$

$S_{x} J f(x, y)=\frac{2}{5} k x^{5}+\frac{1}{6} k(2 n-1) x^{6}+\frac{2}{7} k n x^{7}+\frac{3}{8} k n^{2} x^{8}$,

$S_{x} J D_{x} D_{y}(f(x, y))=\frac{12}{5} k x^{5}+\frac{3}{2} k(2 n-1) x^{6}+\frac{24}{7} k n x^{7}$

$+6 k n^{2} x^{8}$

$S_{x}^{3} Q_{-2} J D_{x}^{3} D_{y}^{3} f(x, y)=\frac{2 \times 6^{3}}{3} k x^{3}+\frac{9^{3}}{4} k(2 n-1) x^{4}$

$+\frac{2 \times 12^{3}}{5} k n x^{5}+\frac{16^{3}}{2} k n^{2} x^{6}$.

Now from Table 1,

1. $M_{1}\left(L\left(C N C_{k}[n]\right)\right)=\left.\left(D_{x}+D_{y}\right)(f(x, y))\right|_{x=y=1}$ $24 \quad 26$

$=24 k n^{2}+26 k n+4 k$.

2. $M_{2}\left(L\left(C N C_{k}[n]\right)\right)=\left.D_{x} D_{y}(f(x, y))\right|_{x=y=1}$

$=48 k n^{2}+42 k n+3 k$.

3. ${ }^{m} M_{2}\left(L\left(C N C_{k}[n]\right)\right)=\left.S_{x} S_{y}(f(x, y))\right|_{x=y=1}$

$=(3 / 16) k n^{2}+(7 / 18) k n+(2 / 9) k$.

4. $R_{\alpha}\left(L\left(C N C_{k}[n]\right)\right)=\left.D_{x}^{\alpha} D_{y}^{\alpha}(f(x, y))\right|_{x=y=1} \quad 3 \quad 16$

$=3 \times 16^{\alpha} k n^{2}+2 \times 9^{\alpha} k n+2 \times 12^{\alpha} k n+2 \times 6^{\alpha} k-9^{\alpha} k$.

5. $R R_{\alpha}\left(L\left(C N C_{k}[n]\right)\right)=\left.S_{x}^{\alpha} S_{y}^{\alpha}(f(x, y))\right|_{x=y=1}$

$=k\left(\frac{3}{16^{\alpha}} n^{2}+\frac{2}{9^{\alpha}} n+\frac{2}{12^{\alpha}} n-\frac{1}{9^{\alpha}}+\frac{2}{6^{\alpha}}\right)$. 
6. $\operatorname{SSD}\left(L\left(C N C_{k}[n]\right)\right)=\left.\left(S_{y} D_{x}+S_{x} D_{y}\right)(f(x, y))\right|_{x=y=1}$ $=6 k n^{2}+\frac{49}{6} k n+\frac{7}{3} k$.

7. $H\left(L\left(C N C_{k}[n]\right)\right)=\left.2 S_{x} J(f(x, y))\right|_{x=1}=\frac{3}{8} k n^{2}$ $+\frac{13}{21} k n+\frac{7}{30} k$

8. $I\left(L\left(C N C_{k}[n]\right)\right)=S_{x} J D_{x} D_{y}(f(x, y))_{x=1}$ $=6 k n^{2}+\frac{45}{7} k n+\frac{9}{10} k$.

9. $A\left(L\left(C N C_{k}[n]\right)\right)=\left.S_{x}^{3} Q_{-2} J D_{x}^{3} D_{y}{ }^{3}(f(x, y))\right|_{x=1}$ 2048

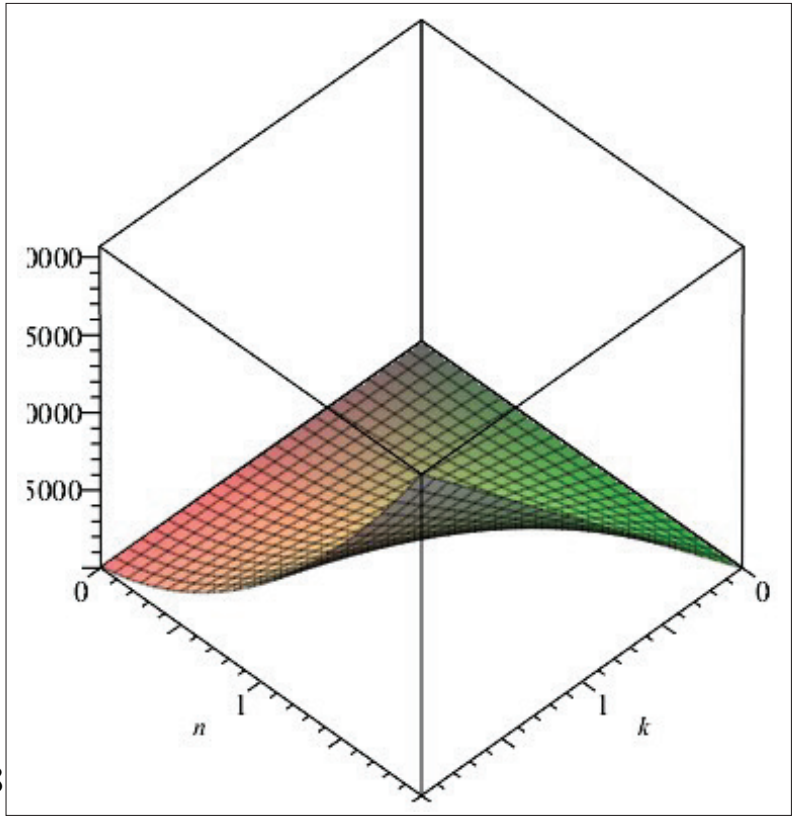

$=2048 k n^{2}+\frac{10557}{10} k n-\frac{153}{4} k$.

Figure 5: 3D plot of augmented Zagreb index

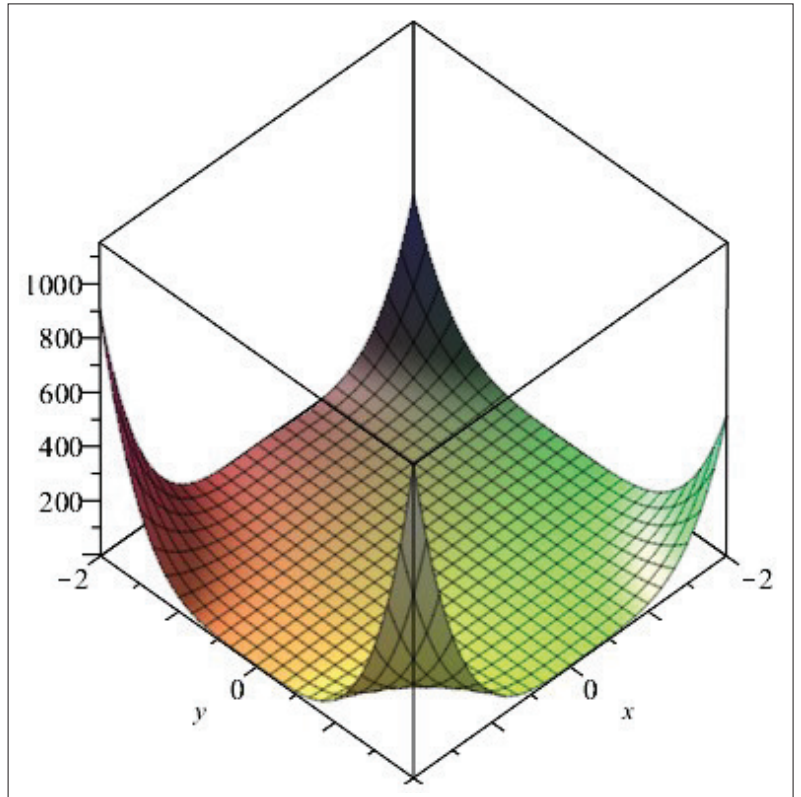

Figure 4: The plot of the M-polynomial of the line graph of carbon nanocones

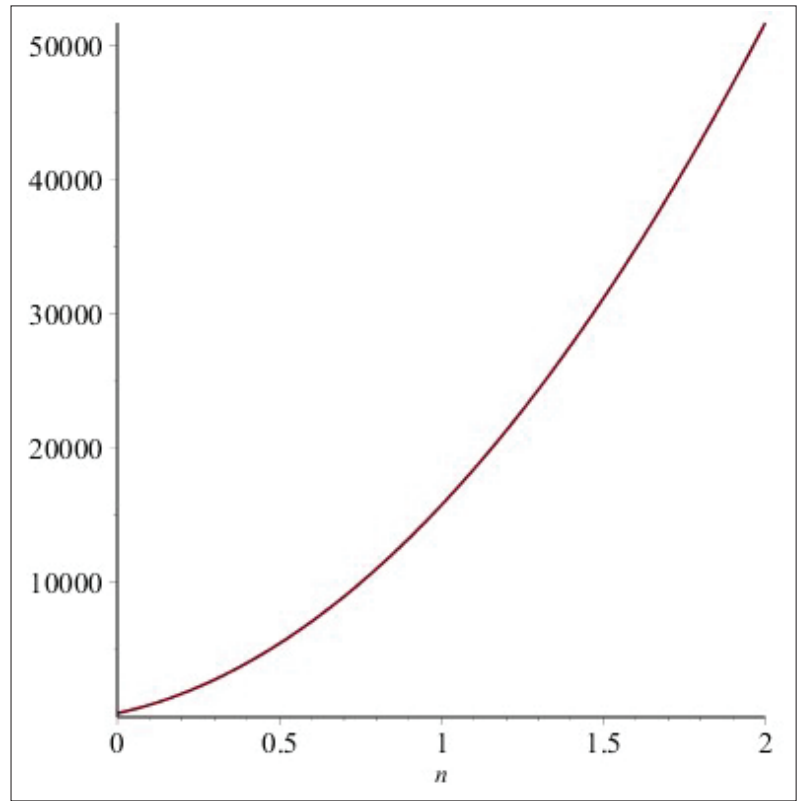

Figure 6: The graph of augmented Zagreb index for $\mathrm{k}=5$ 


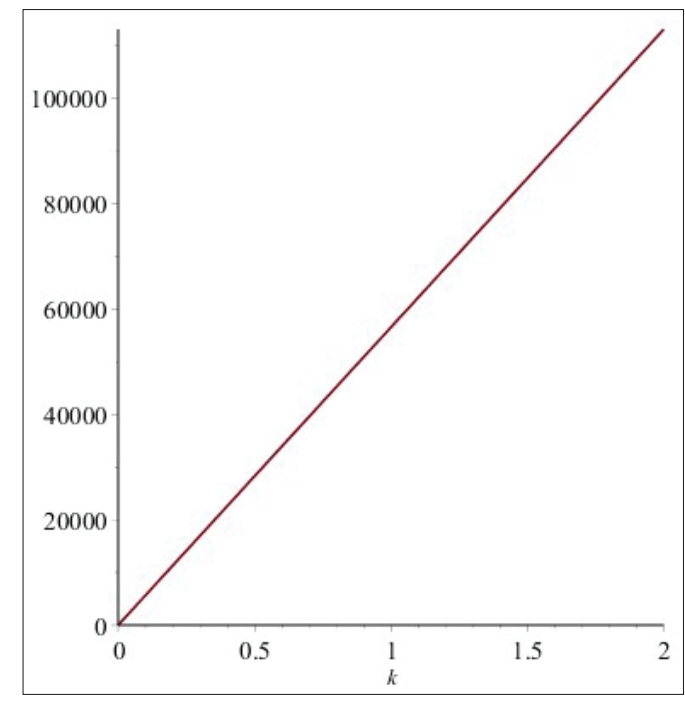

Figure 7: The graph of augmented Zagreb index for $n=5$

\section{CONCLUSION}

In this article, we computed general forms of M-polynomials of $L\left(C N C_{k}[n]\right)$ for all $n$ and $k$. This polynomial gives a thorough insight about topological indices and their dependence on the molecular structure of $L\left(C N C_{k}[n]\right)$. These indices are actually, experimentally correlated with many properties. All topological indices calculated depend similarly on $k$ and $n$. Figure 5 is the surface associated to topological index, whereas Figure 6 shows that topological indices are quadratically related with $n$, but are linearly related with $k$ as Figure 7 suggests.

We pose an open problem to compute the Wiener and other distance-based indices of carbon general nanocones and its line graph. Although some partial results are available for one particular subclass of these nanocones it will be interesting to see how the reactivity is affected by the change in sizes of general nanocones and their Weiner indices.

\section{Acknowledgements}

This research is supported by funds from Dong-A University, South Korea.

\section{REFERENCES}

Adisa O.O., Cox B.J. \& Hill J.M. (2011). Encapsulation of methane molecules into carbon nanotubes. Physica B: Condensed Matter 406(1): 88-93.

DOI: https://doi.org/10.1016/j.physb.2010.10.027
Ali A., Munir M., Nazeer W. \& Kang S.M. (2018). M-polynomials and topological indices of zigza and rhombic benzenoid systems. Open Chemistry 16: 73-78. DOI: https://doi.org/10.1515/chem-2018-0010

Amić D., Bešlo D., Lučić B., Nikolić S. \& Trinajstić N. (1998). The vertex-connectivity index revisited. Journal of Chemical Information and Computer Sciences 38(5): 819-822.

DOI: https://doi.org/10.1021/ci980039b

Ashrafi A.R. \& Mohammad-Abadi Z. (2012). The Wiener index of one-pentagonal carbon nanocone, fullerenes. Nanotubes and Carbon Nanostructures 20(8): 688-695.

DOI: https://doi.org/10.1080/1536383X.2011.552998

Bollobás B. \& Erdös P. (1998). Graphs of extremal weights. Ars Combinatoria 50: 225-233.

Brückler F.M., Došlić T., Graovac A. \& Gutman I. (2011). On a class of distance-based molecular structure descriptors. Chemical Physics Letters 503(4): 336-338. DOI: https://doi.org/10.1016/j.cplett.2011.01.033

Buckley T.F. \& Rapoport H. (1981). Alpha-amino acids as chiral educts for asymmetric products. Amino acylation with n-acylamino acids. Journal of the American Chemical Society 103(20): 6157-6163. DOI: https://doi.org/10.1021/ja00410a030

Caporossi G., Gutman I., Hansen P. \& Pavlović L. (2003). Graphs with maximum connectivity index. Computational Biology and Chemistry 27(1): 85-90. DOI: https://doi.org/10.1016/S0097-8485(02)00016-5

Cataldo F., Ori O. \& Iglesias-Groth S. (2010). Topological lattice descriptors of graphene sheets with fullerene-like nanostructures. Molecular Simulation 36(5): 341-353. DOI: https://doi.org/10.1080/08927020903483262

De N. (2018). Hyper zagreb index of bridge and chain graphs. Open Journal of Mathematical Sciences 2(1): 1 - 17. DOI: https://doi.org/10.30538/oms2018.0013

Deutsch E. \& Klavzar S. (2015). M-Polynomial, and degreebased topological indices. Iranian Journal of Mathematical Chemistry 6: 93-102.

DOI: https://doi.org/10.22052/IJMC.2015.10106

Dobrynin A.A., Entringer R. \& Gutman I. (2001). Wiener index of trees: theory and applications. Acta Applicandae Mathematicae 66(3): 211-249.

DOI: https://doi.org/10.1023/A:1010767517079

Gillot J., Lux B., Cornuault P. \& DuChaffaut F. (1968). Catalysis of the graphitization of a furfuryl alcohol coke by titanium and vanadium. Berichte der Deutschen Keramischen Gesellschaft 45: 224-230.

DOI: https://doi.org/10.1177/026248930802700403

Gutman I. \& Das K.C. (2004). The first Zagreb index 30 years after. Match - Communications in Mathematical and in Computer Chemistry 50: 83-92.

Gutman I. \& Polansky O. E. (1986). Wiener numbers of polyacenes and related benzenoid molecules. MatchCommunications in Mathematical and in Computer Chemistry 20: 115-123.

Gutman I. \& Trinajstić N. (1972). Graph theory and molecular orbitals. Total $\varphi$-electron energy of alternant hydrocarbons. Chemical Physics Letters 17(4): 535-538. DOI: https://doi.org/10.1016/0009-2614(72)85099-1 
Gutman R. (1993). A Witness to Genocide: The 1993 Pulitzer Prize-Winning Dispatches on the" Ethnic Cleansing" of Bosnia. Macmillan Publishing Company, New York, USA.

Hu Y.C., Perrig A. \& Johnson D.B. (2005). Ariadne: A secure on-demand routing protocol for ad hoc networks. Wireless Networks 11(1-2): 21-38.

DOI: https://doi.org/10.1007/s11276-004-4744-y

Huo Y., Qi L., Lv X.J., Lai T., Zhang J. \& Zhang Z.Q. (2016). A sensitive aptasensor for colorimetric detection of adenosine triphosphate based on the protective effect of ATP-aptamer complexes on unmodified gold nanoparticles. Biosensors and Bioelectronics 78: 315-320.

DOI: https://doi.org/10.1016/j.bios.2015.11.043

Iijima S. (1991). Helical microtubules of graphitic carbon. Nature 354: 56-58.

DOI: https://doi.org/10.1038/354056a0

Iranmanesh A., Gutman I., Khormali O. \& Mahmiani A. (2009). The edge versions of the Wiener index. Match Communications in Mathematical and in Computer Chemistry 61(3): 663.

Kier L.B. \& Hall L.H. (1976). Molecular connectivity VII: specific treatment of heteroatoms. Journal of Pharmaceutical Sciences 65(12): 1806-1809. DOI: https://doi.org/10.1002/jps.2600651228

Kier L.B. \& Hall L.H. (1986). Molecular Connectivity in Structure-Activity Analysis. John Wiley and Sons, Inc., New York, USA.

Klavžar S. \& Gutman I. (1996). A Comparison of the Schultz molecular topological index with the Wiener index. The Journal for Chemical Information and Computer Scientists 36: 1001-1003. DOI: https://doi.org/10.1021/ci9603689

Kwun Y.C., Munir M., Nazeer W., Rafique S. \& Kang S.M. (2017). M-Polynomials and topological indices of V-Phenylenic Nanotubes and Nanotori. Scientific Reports 7: 8756 .

DOI: https://doi.org/10.1038/s41598-017-08309-y

Li X., Gutman I. \& Randić M. (2006). Mathematical Aspects of Randić-Type Molecular Structure Descriptors. Faculty of Science, University of Kragujevac, Serbia.

Li X. \& Shi Y. (2008). A survey on the Randic index. Match - Communications in Mathematical and in Computer Chemistry 59(1): 127-156.

Munir M., Nazeer W., Rafique S. \& Kang S.M. (2016a). M-polynomial and related topological indices of nanostar dendrimers. Symmetry 8(9): 97.

DOI: https://doi.org/10.3390/sym8090097

Munir M., Nazeer W., Rafique S., Nizami A.R. \& Kang S.M. (2016b). M-polynomial and degree-based topological indices of titania nanotubes. Symmetry 8(11): 117. DOI: https://doi.org/10.3390/sym8110117

Munir M., Nazeer W., Rafique S. \& Kang S.M. (2016c).
M-polynomial and degree-based topological indices of polyhex nanotubes. Symmetry 8(12): 149.

DOI: https://doi.org/10.3390/sym8120149

Munir M., Nazeer W., Rafique S., Nizami A.R. \& Kang S.M. (2017). Some computational aspects of triangular boron nanotubes. Symmetry 9(1): 6.

DOI: https://doi.org/10.3390/sym9010006

Munir M., Nazeer W., Shahzadi S. \& Kang S.M. (2016). Some invariants of circulant graphs. Symmetry 8(11): 134.

DOI: https://doi.org/10.3390/sym8110134

Nikolić S., Kovačević G., Miličević A. \& Trinajstić N. (2003). The Zagreb indices 30 years after. Croatica Chemica Acta 76(2): 113-124.

Randić M. (1975). Characterization of molecular branching. Journal of the American Chemical Society 97(23): 6609-6615.

DOI: https://doi.org/10.1021/ja00856a001

Randić M. (2001). The connectivity index 25 years after. Journal of Molecular Graphics and Modelling 20(1): 19-35. DOI: https://doi.org/10.1016/S1093-3263(01)00098-5

Randić M. (2008). On history of the Randić index and emerging hostility toward chemical graph theory. Match - Communications in Mathematical and in Computer Chemistry 59: 5-124.

Rehman M.U.H., Sardar R. \& Raza R. (2017). Computing topological indices of Hex board and its line graph. Open Journal of Mathematical Sciences 1(1): 62 - 71.

DOI: https://doi.org/10.30538/oms2017.0007

Rucker G. \& Rucker C. (1999). On topological indices, boiling points, and cycloalkanes. The Journal for Chemical Information and Computer Scientists 39: 788-802.

DOI: https://doi.org/10.1021/ci9900175

Sardar M.S., Zafar S. \& Farahani M.R. (2017). The generalized Zagreb index of Capra-designed planar benzenoid series Cak(C6). Open Journal of Mathematical Sciences 1(1): $44-51$.

DOI: https://doi.org/10.30538/oms2017.0005

Wiener H. (1947). Structural determination of paraffin boiling points. Journal of the American Chemical Society 69(1): 17-20.

DOI: https://doi.org/10.1021/ja01193a005

Wu B. et al. (15 authors) (2010). Structures of the CXCR4 chemokine GPCR with small-molecule and cyclic peptide antagonists. Science 330: 1066-1071.

DOI: https://doi.org/10.1126/science.1194396

Zhao J., Buldum A., Han J. \& Lu J.P. (2002). Gas molecule adsorption in carbon nanotubes and nanotube bundles. Nanotechnology 13(2): 195.

DOI: https://doi.org/10.1088/0957-4484/13/2/312

Zhou B. \& Trinajstić N. (2010). On general sum-connectivity index. Journal of Mathematical Chemistry 47(1): 210-218. DOI: https://doi.org/10.1007/s10910-009-9542-4 